Article

\title{
Rigorous Mathematical Investigation of a Nonlocal and Nonlinear Second-Order Anisotropic Reaction-Diffusion Model: Applications on Image Segmentation
}

\author{
Costi că Moroşanu ${ }^{1, *}$ and Silviu Pavăl ${ }^{2}$ \\ 1 Department of Mathematics, “Alexandru Ioan Cuza” University, Bd. Carol I, 11, 700506 Iaşi, Romania \\ 2 Faculty of Automatic Control and Computer Engineering, Technical University "Gheorghe Asachi” of Iaşi, \\ Dimitrie Mangeron, nr. 27, 700050 Iaşi, Romania; silviu.paval@tuiasi.ro \\ * Correspondence: costica.morosanu@uaic.ro
}

check for updates

Citation: Moroşanu, C.; Pavăl, S. Rigorous Mathematical Investigation of a Nonlocal and Nonlinear SecondOrder Anisotropic Reaction-Diffusion Model: Applications on Image Segmentation. Mathematics 2021, 9, 91. https://doi.org/10.3390/math 9010091

Received: 17 September 2020 Accepted: 29 December 2020 Published: 4 January 2021

Publisher's Note: MDPI stays neutral with regard to jurisdictional claims in published maps and institutional affiliations.

Copyright: () 2021 by the authors. Licensee MDPI, Basel, Switzerland. This article is an open access article distributed under the terms and conditions of the Creative Commons Attribution (CC BY) license (https: / creativecommons.org/ licenses/by/4.0/).

\begin{abstract}
In this paper we are addressing two main topics, as follows. First, a rigorous qualitative study is elaborated for a second-order parabolic problem, equipped with nonlinear anisotropic diffusion and cubic nonlinear reaction, as well as non-homogeneous Cauchy-Neumann boundary conditions. Under certain assumptions on the input data: $f(t, x), w(t, x)$ and $v_{0}(x)$, we prove the well-posedness (the existence, a priori estimates, regularity, uniqueness) of a solution in the Sobolev space $W_{p}^{1,2}(Q)$, facilitating for the present model to be a more complete description of certain classes of physical phenomena. The second topic refers to the construction of two numerical schemes in order to approximate the solution of a particular mathematical model (local and nonlocal case). To illustrate the effectiveness of the new mathematical model, we present some numerical experiments by applying the model to image segmentation tasks.
\end{abstract}

Keywords: nonlinear anisotropic reaction-diffusion; well-posedness of solutions; Leray-Schauder degree theory; finite difference method; explicit numerical approximation scheme; image segmentation

\section{Introduction}

For the unknown function $v(t, x)$ (hereafter, $v$ ), consider the following nonlinear second-order boundary value problem in $Q=(0, T] \times \Omega$, with $T>0$ and a bounded domain $\Omega \subset \mathbb{R}^{2}$ of Lebesgue measures $|\Omega|$, whose boundary $\partial \Omega$ is sufficiently smooth:

$$
\left\{\begin{array}{lc}
p_{1} \frac{\partial}{\partial t} v(t, x)=\Phi\left(v_{x}(t, x)\right)\left[\Psi\left(v_{x}(t, x)\right) \Delta v(t, x)+\nabla \Psi\left(v_{x}(t, x)\right) \cdot \nabla v(t, x)\right] & \\
\quad+p_{3}\left[v(t, x)-v^{3}(t, x)\right]+p_{4} f(t, x) & \text { in } Q \\
\bar{q}(t, x) \frac{\partial}{\partial \mathbf{n}} v(t, x)+p_{5} v(t, x)=w(t, x) & \text { on } \Sigma \\
v(0, x)=v_{0}(x) & \text { on } \Omega
\end{array}\right.
$$

where:

- $t \in(0, T], x=\left(x_{1}, x_{2}\right)$ varies in $\Omega, \Sigma=(0, T] \times \partial \Omega$;

- $\nabla v(t, x)=v_{x}(t, x)\left(\nabla v=v_{x}\right)$ the gradient of $v(t, x)$ in $x$, that is $\nabla v=\left(\frac{\partial}{\partial x_{1}} v, \frac{\partial}{\partial x_{2}} v\right)$.

Setting $\frac{\partial}{\partial x_{i}} v=v_{x_{i}}, i=1,2$, then $\nabla v=\left(v_{x_{1}}, v_{x_{2}}\right)=v_{x}$;

- $\Delta v(t, x)$ is the Laplace operator-a second-order differential operator, defined as the divergence $(\nabla \cdot)$ of the gradient of $v(t, x)$ in $x$;

- $\frac{\partial}{\partial t} v(t, x)$ is the partial derivative of $v(t, x)$ with respect to $t$;

- $p_{1}, p_{3}, p_{4}, p_{5}$ are positive values. 
- $\Phi\left(v_{x}(t, x)\right)$ is a positive and bounded nonlinear real function of class $C^{1}(Q)$ with bounded derivatives (see [1]), having the role of controlling the speed of the diffusion process and enhances the edges (e.g., in the evolving image);

- $\Psi\left(v_{x}(t, x)\right)$ is the mobility;

- $\bar{q}(t, x)$ is a positive and bounded real function;

- $f(t, x) \in L^{p}(Q)$ is the distributed control (a given function), where

$$
p \geq 2
$$

- $\quad w(t, x) \in W_{p}^{1-\frac{1}{2 p}, 2-\frac{1}{p}}(\Sigma)$ is the boundary control (a given function);

- $\quad \mathbf{n}=\mathbf{n}(\mathrm{x})$ is the outward unit normal vector to $\Omega$ at a point $x \in \partial \Omega$. $\frac{\partial}{\partial \mathbf{n}}$ denotes differentiation along $\mathbf{n}$;

- $v_{0}(x) \in W_{p}^{2-\frac{2}{p}}(\Omega)$, verifying

$$
\bar{q}(t, x) \frac{\partial}{\partial \mathbf{n}} v_{0}(x)+p_{5} v_{0}(x)=w(0, x) .
$$

Let us note

$$
a_{i}\left(t, x, v(t, x), v_{x}(t, x)\right)=\Phi\left(v_{x}(t, x)\right) \Psi\left(v_{x}(t, x)\right) v_{x_{i}}(t, x), \quad i=1,2 .
$$

Then, it is easy to recognize Equation $(1)_{1}$ as being quasi-linear with

$$
\begin{aligned}
a_{i j}\left(t, x, v(t, x), v_{x}(t, x)\right)= & \frac{\partial}{\partial v_{x_{j}}} a_{i}\left(t, x, v(t, x), v_{x}(t, x)\right) \\
= & \frac{\partial}{\partial v_{x_{j}}} \Phi\left(v_{x}(t, x)\right) \Psi\left(v_{x}(t, x)\right) v_{x_{i}}(t, x), \quad i=1,2, \\
a\left(t, x, v(t, x), v_{x}(t, x)\right)= & -\frac{\partial}{\partial v}\left(\Phi\left(v_{x}(t, x)\right) \Psi\left(v_{x}(t, x)\right) v_{x_{i}}(t, x)\right) v_{x_{i}}(t, x) \\
& -\frac{\partial}{\partial x_{i}} \Phi\left(v_{x}(t, x)\right) \Psi\left(v_{x}(t, x)\right) v_{x_{i}}(t, x) \\
& -p_{3}\left[v(t, x)-v^{3}(t, x)\right]-p_{4} f(t, x),
\end{aligned}
$$

while the boundary conditions $(1)_{2}$ are of second type:

$$
\left[a_{i j}\left(t, x, v(t, x), v_{x}(t, x)\right) v_{x_{j}}(t, x) \cos \alpha_{i}+p_{5} v(t, x)-w(t, x)\right]_{\Sigma}=0,
$$

(see [1] and reference therein).

For the reader's benefit, we write problem (1) in the equivalent form

$$
\begin{cases}p_{1} \frac{\partial}{\partial t} v(t, x)-\Phi\left(v_{x}(t, x)\right) \operatorname{div}\left(\Psi\left(v_{x}(t, x)\right) \nabla v(t, x)\right) & \\ =p_{3}\left[v(t, x)-v^{3}(t, x)\right]+p_{4} f(t, x) & \text { in } Q \\ \bar{q}(t, x) \frac{\partial}{\partial \mathbf{n}} v(t, x)+p_{5} v(t, x)=w(t, x) & \text { on } \Sigma \\ v(0, x)=v_{0}(x) & \text { on } \Omega .\end{cases}
$$

Concerning Equation (5) 1 , we recall that it is of quasi-linear type with principal part in divergence form (see [1]), with $a_{i}, i=1,2$, given by (4) and 


$$
a\left(t, x, v(t, x), v_{x}(t, x)\right)=-p_{3}\left[v(t, x)-v^{3}(t, x)\right]-p_{4} f(t, x) .
$$

In addition, we assume that Equations (1) $[$ [or (5) 1 ] are uniformly parabolic, i.e.,

$$
v_{1}(|u|) \zeta^{2} \leq a_{i j}(t, x, u, z) \zeta_{i} \zeta_{j} \leq v_{2}(|u|) \zeta^{2}
$$

for arbitrary $u(t, x)$ and $z(t, x),(t, x) \in Q$, and $\zeta=\left(\zeta_{1}, \zeta_{2}\right)$ an arbitrary real vector, where $v_{1}(s), v_{2}(s)$ are positive continuous functions of $s \geq 0, v_{1}(s)$ is nonincreasing and $v_{2}(s)$ is nondecreasing.

The nonlinear problem (1) (or (5)) is important for modeling a variety of phenomena of life sciences, including in biology, biochemistry, economics, medicine and physics. Particular cases of the nonlinear second-order boundary value problem (1), supplied with different boundary conditions, have been successfully applied to many complex moving interface problems, e.g., the motion of anti-phase boundaries in crystalline solids [2], the mixture of two incompressible fluids, the nucleation of solids, and vesicle membranes (see [3-5] and the references therein). In addition, the nonlinear problems of type (1) $)_{1}$, occur in the phase-field transition system (e.g., [6]) where the phase function $v(t, x)$ describes the transition between the solid and liquid phases in the solidification process of a material occupying a region $\Omega$. For more general assumptions and with various types of boundary conditions, Equation (5) has been numerically investigated (e.g., [6-17]). The error analysis for the implicit backward Euler approximation is presented in [16], and computations with several different higher-order time-stepping schemes are used in [11]. For the wellposedness (existence, estimate, uniqueness and regularity) of a solution in Sobolev spaces we refer to [12,18-21].

Another important novelty in our paper concerns the non-homogeneous CauchyNeumann boundary conditions, which can be seen as boundary control in industry. Thus, as applications of problem (1), we indicate the moving interface problems, e.g., phase separation and transition (see $[3,8,12,17,18,22-27]$ ), anisotropy effects (see [15,28-30]), image denoising and segmentation (see $[15,24,26,30-39]$ and references therein), etc.

Definition 1. The function $v(t, x)$ is called a classical solution of the problem (1) if it is continuous in $\bar{Q}$, has continuous derivatives $v_{t}, v_{x}, v_{x x}$ in $Q$, verifies $(1)_{1}$ in every $(t, x) \in Q$ and verifies $(1)_{2}$ and $(1)_{3}$ for $(t, x) \in \Sigma$ and $t=0$, respectively.

In our paper, we study the solvability of the problems (1) in the class $W_{p}^{1,2}(Q)$, characterized by the presence of some new physical parameters $\left(p_{1}, p_{3}, p_{4}, p_{5}, \Phi\left(v_{x}(t, x)\right)\right.$, $\left.\Psi\left(v_{x}(t, x)\right)\right)$, the principal part being in divergence form and by considering the cubic nonlinearity $p_{3}\left[v(t, x)-v^{3}(t, x)\right]$, satisfying for $n \in\{1,2,3\}$ the assumption $H_{0}$ in [21], that is:

$H_{0}:\left(v-v^{3}\right)|v|^{3 p-4} v \leq 1+|v|^{3 p-1}-|v|^{3 p}$.

In Theorem 1, we prove the existence, regularity and uniqueness of solution for (1). (see [15] for a numerical study of Equation (1) corresponding to a linear reaction term $v(t, x)-v_{0}(x)$, with homogeneous Neumann boundary condition).

In the following we will denote by $C$ several positive constants.

\section{Well-Posedness of the Solution of (5)}

Theorem 1 of this section presents the dependence of the solution $v(t, x)$ of (5) on $f(t, x)$ and $w(t, x)$. In our study, we rely on the following:

- $\quad$ The Leray-Schauder principle (see [1,4,11-15,19-21] and reference therein);

- The $L^{p}$-theory of linear and quasi-linear parabolic equations;

- Green's first identity

$$
-\int_{\Omega} y \operatorname{div} z d x=\int_{\Omega} \nabla y \cdot z d x-\int_{\partial \Omega} y \frac{\partial}{\partial \mathbf{n}} z d \gamma,
$$




$$
-\int_{\Omega} y \Delta z d x=\int_{\Omega} \nabla y \cdot \nabla z d x-\int_{\partial \Omega} y \frac{\partial}{\partial \mathbf{n}} z d \gamma,
$$

for any scalar-valued function $y$ and $z$, a continuously differentiable vector field in $n$ dimensional space;

- $\quad$ The Lions and Peetre embedding theorem (see [1] and references therein) to ensure the existence of a continuous embedding $W_{p}^{1,2}(Q) \subset L^{\mu}(Q)$, where the number $\mu$ is defined as follows (see (3))

$$
\mu= \begin{cases}\text { any positive number } \geq 3 p & \text { if } \quad p \geq 2, \\ \left(\frac{1}{p}-\frac{1}{2}\right)^{-1}, & \text { if } \quad p<2 .\end{cases}
$$

and, for $k \in\{1,2, \ldots\}$ and $1 \leq p \leq \infty, W_{p}^{k, 2 k}(Q)$ denotes the Sobolev space on $Q$ :

$$
W_{p}^{k, 2 k}(Q)=\left\{y \in L^{p}(Q): \frac{\partial^{r}}{\partial t^{r}} \frac{\partial^{q}}{\partial x^{q}} y \in L^{p}(Q), \text { for } 2 r+q \leq 2 k\right\},
$$

(see [1] for more details).

In addition, we use the set $C^{1,2}(\bar{Q})\left(C^{1,2}(Q)\right)$ of all continuous functions in $\bar{Q}$ (in $Q$ ) having continuous derivatives $u_{t}, u_{x}$ and $u_{x x}$ in $\bar{Q}$ (in $Q$ ), as well as the Sobolev spaces $W_{p}^{l}(\Omega), W_{p}^{l, l / 2}(\Sigma)$ with non-integral $l$ for the initial and boundary conditions, respectively (see [1]).

The main result for the study of the existence, a priori estimates, uniqueness and regularity for the solution of (1) (or (5)) is the next theorem.

Theorem 1. For any classical solution $v(t, x) \in C^{1,2}(Q)$ of $(5)$, suppose there are $M, M_{0}, m_{1}$, $M_{1}, M_{2}, M_{3}$ and $M_{4} \in(0, \infty)$ such that the fpllowing hypotheses are satisfied:

$\mathbf{I}_{1}$. $|v(t, x)|<M$ for any $(t, x) \in Q$ and for any $z(t, x)$, the map $\Psi(z(t, x))$ is continuous, differentiable in $x$, its $x$-derivatives are measurable bounded, satisfies (6) and

$$
\begin{gathered}
0<\Psi_{\text {min }} \leq \Psi\left(v_{x}(t, x)\right)<\Psi_{\text {max }}, \quad \text { for }(t, x) \in Q, \\
\left|\Psi(z) v_{x_{i}}\right|(1+|z|)+\left|\frac{\partial}{\partial x_{1}}\left(\Psi(z) v_{x_{1}}\right)\right|+\left|\frac{\partial}{\partial x_{2}}\left(\Psi(z) v_{x_{1}}\right)\right| \\
+\left|\frac{\partial}{\partial x_{1}}\left(\Psi(z) v_{x_{2}}\right)\right|+\left|\frac{\partial}{\partial x_{2}}\left(\Psi(z) v_{x_{2}}\right)\right|+\left|v\left(t, x_{1}, x_{2}\right)\right| \leq M_{0}(1+|z|)^{2} .
\end{gathered}
$$

$\mathbf{I}_{2} . \Phi\left(v_{x}(t, x)\right)$ is a positive and bounded nonlinear real function of class $C^{1}(Q)$ with bounded derivatives and

$$
0<m_{1} \leq \Phi\left(v_{x}(t, x)\right) \leq M_{1} .
$$

In addition, for every $\varepsilon>0$, the functions $v(t, x)$ and $\Psi\left(v_{x}(t, x)\right)$ satisfy the relations

$$
\|v\|_{L^{s}(Q)} \leq M_{2^{\prime}} \quad\left\|\Psi\left(v_{x}\right) v_{x_{i}}\right\|_{L^{r}(Q)}<M_{3^{\prime}}, \quad i=1,2,
$$

where

$$
r=\left\{\begin{array}{ll}
\max \{p, 4\} & p \neq 4 \\
4+\varepsilon & p=4,
\end{array} \quad s= \begin{cases}\max \{p, 2\} & p \neq 2 \\
2+\varepsilon & p=2\end{cases}\right.
$$


Then, $\forall f \in L^{p}(Q)$ and $\forall v_{0} \in W_{p}^{2-\frac{2}{p}}(\Omega)$, with $p \neq \frac{3}{2}$, the problem (5) has a solution $v \in W_{p}^{1,2}(Q)$ and the next estimate holds:

$$
\begin{aligned}
& \|v\|_{W_{p}^{1,2}(Q)} \leq C\left[1+\left\|v_{0}\right\|_{W_{p}^{2-\frac{2}{p}}(\Omega)}+\left\|v_{0}\right\|_{L^{3 p-2}(\Omega)}^{3-\frac{2}{p}}\right. \\
& \left.+\|f\|_{L^{p}(Q)}+\|w\|_{L^{3 p-2}(\Sigma)}^{3-\frac{2}{p}}+\|w\|_{W_{p}^{1-\frac{1}{2 p}, 2-\frac{1}{p}}(\Sigma)}\right],
\end{aligned}
$$

where the constant $C>0$ does not depend on $v, f$ and $w$.

If $v^{1}, v^{2} \in W_{p}^{1,2}(Q)$ are two solutions to (5), corresponding to $\left\{f^{1}, w^{1}, v_{0}^{1}\right\}$ and $\left\{f^{2}, w^{2}, v_{0}^{2}\right\}$, respectively, such that $\left\|v^{1}\right\|_{W_{p}^{1,2}(Q)} \leq M_{4},\left\|v^{2}\right\|_{W_{p}^{1,2}(Q)} \leq M_{4}$ and

$$
0<q_{\min } \leq \bar{q}(t, x)<q_{\max }, \quad \text { for }(t, x) \in \Sigma,
$$

then the following estimate holds:

$$
\max _{(t, x) \in Q}\left|v^{1}-v^{2}\right| \leq C_{1} e^{C T} \max \left[\max _{(t, x) \in Q}\left|f^{1}-f^{2}\right|, \underset{(t, x) \in \Sigma}{\max }\left|w^{1}-w^{2}\right|, \underset{(t, x) \in \Omega}{\max }\left|v_{0}^{1}-v_{0}^{2}\right|\right],
$$

where the constant $C, C_{1}>0$ does not depend on $\left\{v^{1}, f^{1}, w^{1}, v_{0}^{1}\right\}$ and $\left\{v^{2}, f^{2}, w^{2}, v_{0}^{2}\right\}$. In particular, the solution of problem (5) is unique.

\subsection{The Proof of Theorem 1}

To prove this theorem, we use the Leray-Schauder principle. Thus, we consider the Banach space

$$
B=W_{p}^{0,1}(Q) \cap L^{3 p}(Q),
$$

endowed with the norm

$$
\|u\|_{B}=\|u\|_{L^{p}(Q)}+\left\|u_{x}\right\|_{L^{p}(Q)},
$$

and a nonlinear operator $H: B \times[0,1] \rightarrow B$ defined by

$$
v=v(u, \lambda)=H(u, \lambda) \quad \text { for all } \quad(u, \lambda) \in W_{p}^{0,1}(Q) \cap L^{3 p}(Q) \times[0,1],
$$

where $v(u, \lambda)$ is the unique solution to the next problem

$$
\begin{cases}p_{1} \frac{\partial}{\partial t} v(t, x)-\left[\lambda \Phi\left(u_{x}(t, x)\right) \frac{\partial}{\partial u_{x_{j}}}\left(\Psi\left(u_{x}\right) u_{x_{i}}\right)+(1-\lambda) \delta_{i}^{j}\right] v_{x_{i} x_{j}} & \\ =\lambda\left\{A\left(t, x, u, u_{x}\right)+p_{3}\left[u(t, x)-u^{3}(t, x)\right]+p_{4} f(t, x)\right\} & \text { in } Q \\ \bar{q}(t, x) \frac{\partial}{\partial v} v(t, x)+p_{5} v(t, x)=\lambda w(t, x) & \text { on } \Sigma \\ v(0, x)=\lambda v_{0}(x), & \text { on } \Omega .\end{cases}
$$

with $A\left(t, x, u, u_{x}\right)=\Phi\left(u_{x}\right) \nabla \Psi\left(u_{x}\right) \cdot \nabla u, \forall(t, x) \in Q$.

We shall prove now the following technical lemma

Lemma 1. We assume Hypotheses $I_{1}$ and $I_{2}$ to be valid. Then

$$
A\left(t, x, u, u_{x_{i}}\right)+p_{3}\left(u-u^{3}\right)+p_{4} f(t, x) \in L^{p}(Q), \quad \forall u \in W_{p}^{0,1}(Q) \cap L^{3 p}(Q) .
$$


Proof. Indeed, since $u \in L^{3 p}(Q)$, then $\|u\|_{L^{3 p}(Q)} \leq$ Konst and thus

$$
\left\|u^{3}\right\|_{L^{p}(Q)}=\left(\int_{Q}\left|u^{3}\right|^{p} d x d t\right)^{\frac{1}{p}}=\left[\left(\int_{Q}|u|^{3 p} d x d t\right)^{\frac{1}{3 p}}\right]^{3 p \frac{1}{p}}=\|u\|_{L^{3 p}(Q)}^{3} \leq(\text { Konst })^{3},
$$

i.e., the nonlinear term in (16) belongs to $L^{p}(Q), \forall u \in W_{p}^{0,1}(Q) \cap L^{3 p}(Q)$ (see also [1]).

Next, from (10) it is easy to conclude that

$$
\frac{\partial}{\partial x_{i}}\left[\Psi\left(u_{x_{i}}\right) u_{x_{i}}\right] \leq M_{1}\left(1+\left|u_{x_{i}}\right|\right)^{2} .
$$

Thus, to prove that

$$
\begin{aligned}
& \left.A\left(t, x, u, u_{x_{i}}\right)\right)=\Phi\left(u_{x}(t, x)\right) \nabla \Psi\left(u_{x}(t, x)\right) \cdot \nabla u(t, x) \in L^{p}(Q), \\
& \forall u \in W_{p}^{0,1}(Q) \cap L^{3 p}(Q),
\end{aligned}
$$

we have to prove that $u_{x_{i}}^{2} \in L^{p}(Q), \forall u \in W_{p}^{0,1}(Q) \cap L^{3 p}(Q)$. For any $u \in W_{p}^{0,1}(Q) \cap L^{3 p}(Q)$ it follows that $\|u\|_{L^{p}(Q)}+\left\|u_{x}\right\|_{L^{p}(Q)} \leq$ konst, i.e., $\left\|u_{x}\right\|_{L^{p}(Q)} \leq$ konst. Making use of the boundedness of $\Phi\left(u_{x}(t, x)\right)$ (see $\left.\mathrm{I}_{2}\right)$, as well as the properties of $\Psi\left(u_{x}(t, x)\right)$ (see $\mathrm{I}_{1}$ ), and since $u_{x_{i}} \in L^{p}(Q)$, it results that $A\left(t, x, u, u_{x_{i}}\right) \in L^{p}(Q), \forall u \in W_{p}^{0,1}(Q) \cap L^{3 p}(Q)$.

Finally, we recall that $f(t, x) \in L^{p}(Q)$ and, owing to the above, we easy derive that the statement expressed by (16) is true.

\subsection{The Proof of Theorem 1 (Continued)}

Let us show that the nonlinear operator $H(u, \lambda)$ defined by (14) satisfies the following Properties A and B.

A. If (15) has a unique solution, then $H$ is well-defined. By the right hand of $(15)_{1}$, using Lemma 1, it follows that, $\forall u \in W_{p}^{0,1}(Q) \cap L^{3 p}(Q)$, then $A\left(t, x, u, u_{x}\right)+p_{3}\left(u-u^{3}\right)+$ $p_{4} f(t, x) \in L^{p}(Q)$ and thus, the same reasoning as in [1] allows us to conclude that for $w(t, x) \in W_{p}^{2-\frac{1}{p}, 1-\frac{1}{2 p}}(\Sigma)$, the linear parabolic boundary value problem formulated in (15) has a unique solution, that is (see (14)) $v=H(u, \lambda) \in W_{p}^{1,2}(Q), \forall u \in B$ and $\forall \lambda \in[0,1]$. Next, the embedding $W_{p}^{1,2}(Q) \subset L^{\mu}(Q) \subset L^{3 p}(Q), p \geq 2$ (see (3) and (7)), allows us to conclude that

$$
H(u, \lambda)=v \in B, \forall u \in B \text { and } \forall \lambda \in[0,1] .
$$

Thus, the operator $H$ is well-defined.

B. Let us now show that $H$ is continuous and compact. The sketch of the proof is the same as in $[1,15]$. However, for reader convenience, we present details in the sequel. Let $u^{n} \rightarrow u$ in $W_{p}^{0,1}(Q) \cap L^{3 p}(Q)$ and $\lambda_{n} \rightarrow \lambda$ in $[0,1]$. Making the notation

$$
v^{n, \lambda_{n}}=H\left(u^{n}, \lambda_{n}\right), \quad v^{n, \lambda}=H\left(u^{n}, \lambda\right) \quad \text { and } \quad v^{\lambda}=H(u, \lambda)
$$

and then considering the difference $H\left(u^{n}, \lambda_{n}\right)-H\left(u^{n}, \lambda\right)$, we obtain from relations (14) and (15) that

$$
\begin{cases}p_{1} \frac{\partial}{\partial t} V^{n, \lambda_{n}, \lambda}-\left[\lambda \Phi\left(u_{x}^{n}\right) \frac{\partial}{\partial u_{x_{j}}^{n}}\left(\Psi\left(u_{x}^{n}\right) u_{x_{i}}^{n}\right)+(1-\lambda) \delta_{i}^{j}\right] V_{x_{i} x_{j}}^{n, \lambda_{n}, \lambda} & \\ =\left(\lambda_{n}-\lambda\right)\left\{\left[\Phi\left(u_{x}^{n}\right) \frac{\partial}{\partial u_{x_{j}}^{n}}\left(\Psi\left(u_{x}^{n}\right) u_{x_{i}}^{n}\right)-\delta_{i}^{j}\right] v_{x_{i} x_{j}}^{n, \lambda_{n}}\right. & \\ \left.\quad+A\left(t, x, u^{n}, u_{x_{i}}^{n}\right)+p_{3}\left[u^{n}-\left(u^{n}\right)^{3}\right]+p_{4} f(t, x)\right\} & \text { in } Q \\ \bar{q}(t, x) \frac{\partial}{\partial \mathbf{n}} V^{n, \lambda_{n}, \lambda}+p_{5} V^{n, \lambda_{n}, \lambda}=\left(\lambda_{n}-\lambda\right) w(t, x) & \text { on } \Sigma \\ v(0, x)=\left(\lambda_{n}-\lambda\right) v_{0}(x) & \text { on } \Omega,\end{cases}
$$


where $V^{n, \lambda_{n}, \lambda}=v^{n, \lambda_{n}}-v^{n, \lambda}$.

The right-hand side in (17) belongs to $L^{p}(Q)$, since $v^{n, \lambda_{n}} \in W_{p}^{1,2}(Q)$. Therefore, the $L^{p}$-theory of PDE gives the estimate

$$
\begin{gathered}
\left\|V^{n, \lambda_{n}, \lambda}\right\|_{W_{p}^{1,2}(Q)} \leq C\left|\lambda_{n}-\lambda\right| \times\left\{\left\|\left[\Phi\left(u_{x}^{n}\right) \frac{\partial}{\partial u_{x_{j}}^{n}}\left(\Psi\left(u_{x}^{n}\right) u_{x_{i}}^{n}\right)-\delta_{i}^{j}\right] v_{x_{i} x_{j}}^{n, \lambda_{n}}\right\|_{L^{p}(Q)}\right. \\
+\left\|A\left(t, x, u^{n}, u_{x_{i}}^{n}\right)\right\|_{L^{p}(Q)}+\left\|u^{n}-\left(u^{n}\right)^{3}\right\|_{L^{p}(Q)} \\
\left.+\left\|v_{0}\right\|_{W_{p}^{2-\frac{2}{p}}(\Omega)}+\|f\|_{L^{p}(Q)}+\|w\|_{W_{p}^{1-\frac{1}{2 p}, 2-\frac{1}{p}}(\Sigma)}\right\}
\end{gathered}
$$

with a constant $C\left(|\Omega|, p_{1}, p_{3}, p_{4}, M, M_{1}, M_{2}, M_{3}\right)$.

Owing to Lemma 1 we can derive that $\left(u^{n}\right)^{3}$ is bounded in $L^{p}(Q), \forall u^{n} \in W_{p}^{0,1}(Q) \cap$ $L^{3 p}(Q)$. In addition, the inequality (10), the working Hypothesis $I_{2}$ and the inclusion $u_{x_{i} x_{j}}^{n, \lambda_{n}} \in L^{p}(Q)$, imply the boundedness in $L^{p}(Q)$ of the terms $A\left(t, x, u^{n}, u_{x_{i}}^{n}\right)$ and $\left(\Phi\left(u_{x}^{n}\right) \frac{\partial}{\partial u_{x_{j}}^{n}}\left(\Psi\left(u_{x}^{n}\right) u_{x_{i}}^{n}\right)-\delta_{i}^{j}\right) v_{x_{i} x_{j}}^{n, \lambda_{n}}$. Moreover, since $W_{p}^{2-\frac{2}{p}}(\Omega) \subset L^{p}(\Omega)$, it results that the remaining terms on the right-hand side from the above inequality are also bounded in $L^{p}(Q)$. Thus, making $\lambda_{n} \rightarrow \lambda$, we obtain $\left(V^{n, \lambda_{n}, \lambda}=v^{n, \lambda_{n}}-v^{n, \lambda}\right)$

$$
\left\|v^{n, \lambda_{n}}-v^{n, \lambda}\right\|_{W_{p}^{1,2}(Q)} \rightarrow 0 \text { for } n \rightarrow \infty .
$$

To evaluate the difference $H\left(v^{n}, \lambda\right)-H(v, \lambda)$, we use again the relations (14), (15), and we obtain

$$
\begin{cases}p_{1} \frac{\partial}{\partial t} V^{n, 1, \lambda}-\left[\lambda \Phi\left(u_{x}^{n}\right) \frac{\partial}{\partial u_{x_{j}}^{n}}\left(\Psi\left(u_{x}^{n}\right) u_{x_{i}}^{n}\right)+(1-\lambda) \delta_{i}^{j}\right] V_{x_{i} x_{j}}^{n, 1, \lambda} & \\ =\lambda\left\{\left[\Phi\left(u_{x}^{n}\right) \frac{\partial}{\partial u_{x_{j}}^{n}}\left(\Psi\left(u_{x}^{n}\right) u_{x_{i}}^{n}\right)-\Phi\left(u_{x}\right) \frac{\partial}{\partial u_{x_{j}}}\left(\Psi\left(u_{x}\right) u_{x_{i}}\right)\right] v_{x_{i} x_{j}}^{\lambda}\right. & \\ \left.+A\left(t, x, u^{n}, u_{x_{i}}^{n}\right)-A\left(t, x, u, u_{x_{i}}\right)+p_{3}\left[\left(u^{n}-u\right)-\left(\left(u^{n}\right)^{3}-u^{3}\right)\right]\right\} & \text { in } Q \\ \bar{q}(t, x) \frac{\partial}{\partial \mathbf{n}} V^{n, 1, \lambda}+p_{5} V^{n, 1, \lambda}=0 & \text { on } \Sigma \\ V^{n, 1, \lambda}(0, x)=0 & \text { on } \Omega,\end{cases}
$$

where $V^{n, 1, \lambda}=v^{n, \lambda}-v^{\lambda}$.

The $L^{p}$-theory applied to (19), gives us the estimate

$$
\begin{aligned}
\left\|V^{n, 1, \lambda}\right\|_{W_{p}^{1,2}(Q) \leq C} \leq & \lambda\left[\left\|\left(\Phi\left(u_{x}^{n}\right) \frac{\partial}{\partial u_{x_{j}}^{n}}\left(\Psi\left(u_{x}^{n}\right) u_{x_{i}}^{n}\right)-\Phi\left(u_{x}\right) \frac{\partial}{\partial u_{x_{j}}}\left(\Psi\left(u_{x}\right) u_{x_{i}}\right)\right) v_{x_{i} x_{j}}^{\lambda}\right\|_{L^{p}(Q)}\right. \\
& +\left\|A\left(t, x, u^{n}, u_{x_{i}}^{n}\right)-A\left(t, x, u, u_{x_{i}}\right)\right\|_{L^{p}(Q)} \\
& \left.+\left\|\left(u^{n}-u\right)-\left(\left(u^{n}\right)^{3}-u^{3}\right)\right\|_{\left.L^{p}(Q)\right]}\right]
\end{aligned}
$$


with a new constant $C$. From the convergence $u^{n} \rightarrow u$ in $W_{p}^{0,1}(Q) \cap L^{3 p}(Q)$ and the continuity of the Nemytskij operator (see [19] and references therein), as well as the continuity of $\Phi\left(u_{x}^{n}\right), \frac{\partial}{\partial u_{x_{j}}^{n}}\left(\Psi\left(u_{x_{i}}^{n}\right) u_{x_{i}}^{n}\right)$ and $A\left(t, x, u^{n}, u_{x_{i}}^{n}\right)$, it follows that

$$
\left\|v^{n, \lambda}-v^{\lambda}\right\|_{W_{p}^{1,2}(Q)} \rightarrow 0 \text { as } n \rightarrow \infty .
$$

Making use of the relations (18) and (20), we show the continuity of the nonlinear operator $H$ defined by (14). Moreover, $H$ is compact. Indeed, since $\mu>3 p$, the inclusion $W_{p}^{1,2}(Q) \hookrightarrow W_{p}^{0,1}(Q) \cap L^{3 p}(Q)$ is compact (see [12] and reference therein). Furthermore, writing $H$ as the composition

$$
B \times[0,1] \rightarrow W_{p}^{1,2}(Q) \hookrightarrow W_{p}^{0,1}(Q) \cap L^{3 p}(Q)=B,
$$

the compactness of $H$ immediately follows.

2.2.1. The Proof of the First Part in Theorem 1: The Regularity of $v(t, x)$

We establish now the existence of a number $\delta>0$ such that

$$
(v, \lambda) \in W_{p}^{0,1}(Q) \cap L^{3 p}(Q) \times[0,1] \text { with } v=H(v, \lambda) \Longrightarrow\|v\|_{B}<\delta .
$$

The equality $v=H(v, \lambda)$ in (21) is equivalent to

$$
\begin{cases}p_{1} \frac{\partial}{\partial t} v(t, x)-\lambda \Phi\left(v_{x}\right) \operatorname{div}\left(\Psi\left(v_{x}\right) \nabla v\right)-(1-\lambda) \Delta v & \\ =\lambda\left[p_{3}\left(v(t, x)-v^{3}(t, x)\right)+p_{4} f(t, x)\right] & \text { in } Q \\ \bar{q}(t, x) \frac{\partial}{\partial \mathbf{n}} v(t, x)+p_{5} v(t, x)=\lambda w(t, x) & \text { on } \Sigma \\ v(0, x)=\lambda v_{0}(x) & \text { on } \Omega .\end{cases}
$$

(see (4), (6) and (15))

Multiplying the first equation in (22) by $|v|^{3 p-4} v$, integrating over $Q_{t}:=(0, t) \times \Omega$, $t \in(0, T]$, we get

$$
\begin{aligned}
& p_{1} \int_{Q_{t}} \frac{\partial}{\partial t}|v(\tau, x)|^{3 p-2} d \tau d x-\lambda \int_{Q_{t}} \Phi\left(v_{x}\right) \operatorname{div}\left(\Psi\left(v_{x}\right) \nabla v\right)|v|^{3 p-4} v d \tau d x \\
& -(1-\lambda) \iint_{Q_{t}} \Delta v|v|^{3 p-4} v d \tau d x \\
& =\lambda p_{3} \int_{Q_{t}}\left(v-v^{3}\right)|v|^{3 p-4} v d \tau d x+\lambda p_{4} \int_{Q_{t}} f|v|^{3 p-4} v d \tau d x .
\end{aligned}
$$


Owing to Green's first identity, the left inequality in (9) and (12), Assumption $I_{2}$ and the boundary conditions $(22)_{2}$, the previous equality leads us to

$$
\begin{aligned}
& \frac{p_{1}}{3 p-2} \int_{\Omega}|v(t, x)|^{3 p-2} d x+\lambda \int_{Q_{t}} \Psi\left(v_{x}\right) \nabla v \cdot \nabla\left(\Phi\left(v_{x}\right)|v|^{3 p-4} v\right) d \tau d x \\
& \quad+(1-\lambda)(3 p-3) \int_{Q_{t}}|\nabla v|^{2}|v|^{3 p-4} d \tau d x \\
& +\lambda p_{5} m_{1} \int_{\Sigma_{t}}|v|^{3 p-2} d \tau d \gamma+\frac{(1-\lambda)}{q_{\max }} p_{5} \int_{\Sigma_{t}}|v|^{3 p-2} d \tau d \gamma \\
& \leq \lambda \frac{p_{1}}{3 p-2} \int_{\Omega}\left|v_{0}(x)\right|^{3 p-2} d x \\
& +\lambda p_{3} \int_{Q_{t}}\left(v-v^{3}\right)|v|^{3 p-4} v d \tau d x+\lambda p_{4} \int_{Q_{t}} f|v|^{3 p-4} v d \tau d x \\
& \quad+\lambda M_{1} \int_{\Sigma_{t}} w|v|^{3 p-4} v d \tau d \gamma+\frac{(1-\lambda)}{q_{\min }} \int_{\Sigma_{t}} w|v|^{3 p-4} v d \tau d \gamma .
\end{aligned}
$$

for all $t \in(0, T]$. The Hölder and Cauchy inequalities, applied to the last terms in (23), give us

$$
\begin{aligned}
& \mathbf{i}_{1} \lambda p_{4} \int_{Q_{t}} f|v|^{3 p-4} v d \tau d x \leq \frac{p-1}{p} \varepsilon^{\frac{p}{p-1}} \int_{Q_{t}}|v|^{3 p} d \tau d x+\lambda p_{4} \frac{1}{p} \varepsilon^{-p}\|f\|_{L^{p}(Q)}^{p} \\
& \mathbf{i}_{2} \lambda M_{1} \int_{\Sigma_{t}} w|v|^{3 p-4} v d \tau d \gamma \\
& \quad \leq \lambda p_{5} m_{1}\left(1-\frac{1}{3 p-2}\right) \int_{\Sigma_{t}}|v|^{3 p-2} d \tau d \gamma+\frac{M_{1}}{m_{1}} \frac{1}{p_{5}} \frac{1}{3 p-2} \int_{\Sigma_{t}}|w|^{p} d \tau d \gamma, \\
& \mathbf{i}_{3} \frac{(1-\lambda)}{q_{\min }} \int_{\Sigma_{t}} w|v|^{3 p-4} v d \tau d \gamma \\
& \quad \leq p_{5}\left(1-\frac{1}{3 p-2}\right) \frac{(1-\lambda)}{q_{\max }} \int_{\Sigma_{t}}|v|^{3 p-2} d \tau d \gamma+\frac{q_{\max }}{q_{\min }} \frac{1}{p_{5}} \frac{1}{3 p-2} \int_{\Sigma_{t}}|w|^{3 p-2} d \tau d \gamma .
\end{aligned}
$$

By $\mathrm{H}_{0}$, relation (3) and Young's inequality, we obtain

$$
\begin{aligned}
& \lambda p_{3} \int_{Q_{t}}\left(v-v^{3}\right)|v|^{3 p-4} v d \tau d x \\
& \quad \leq \lambda p_{3}|\Omega| T+\lambda p_{3}|\Omega| T \frac{1}{3 p} \varepsilon^{-3 p}+\frac{3 p-1}{3 p} \varepsilon^{\frac{3 p}{3 p-1}} \int_{Q_{t}}|v|^{3 p} d \tau d x \\
& \quad-\lambda p_{3} \int_{Q_{t}}|v|^{3 p} d \tau d x .
\end{aligned}
$$


Owing to the above inequality as well as $\left(\mathbf{i}_{1}-\mathbf{i}_{3}\right)$ and, taking into account the continuous embedding $L^{3 p-2}\left(\Sigma_{t}\right) \subset L^{p}\left(\Sigma_{t}\right)$, from (23), we derive the following estimate

$$
\begin{aligned}
& \frac{p_{1}}{3 p-2} \int_{\Omega}|v(t, x)|^{3 p-2} d x+\lambda \int_{Q_{t}} \Psi\left(v_{x}\right) \nabla v \cdot \nabla\left(\Phi\left(v_{x}\right)|v|^{3 p-4} v\right) d \tau d x \\
& \quad+(1-\lambda)(3 p-3) \int_{Q_{t}}|\nabla v|^{2}|v|^{3 p-4} d \tau d x+\lambda p_{3} \int_{Q_{t}}|v|^{3 p} d \tau d x \\
& \quad+p_{5} \frac{1}{3 p-2}\left[\lambda m_{1}+\frac{(1-\lambda)}{q_{\max }}\right] \int_{\Sigma_{t}}|v|^{3 p-2} d \tau d \gamma \\
& \leq \lambda \frac{p_{1}}{3 p-2} \int_{\Omega}\left|v_{0}(x)\right|^{3 p-2} d x \\
& \quad+\left[\frac{3 p-1}{3 p} \varepsilon^{\frac{3 p}{3 p-1}}+\frac{p-1}{p} \varepsilon^{\frac{p}{p-1}}\right] \int_{Q_{t}}|v|^{3 p} d \tau d x \\
& \quad+\lambda\left(p_{3}|\Omega| T+p_{3}|\Omega| T \frac{1}{3 p} \varepsilon^{-3 p}+p_{4} \frac{1}{p} \varepsilon^{-p}\|f\|_{L^{p}(Q)}^{p}\right) \\
& \quad+\frac{1}{p_{5}} \frac{1}{3 p-2}\left[\frac{M_{1}}{m_{1}}+\frac{q_{\max }}{q_{\min }}\right] \int_{\Sigma_{t}}|w|^{3 p-2} d \tau d \gamma .
\end{aligned}
$$

Taking $\varepsilon$ small enough, the previous inequality yields

$$
\lambda\left\||v|^{3}\right\|_{L^{p}(Q)}^{p} \leq C_{1}\left(1+\left\|v_{0}\right\|_{L^{3 p-2}(\Omega)}^{3 p-2}+\|f\|_{L^{p}(Q)}^{p}+\|w\|_{L^{3 p-2}\left(\Sigma_{t}\right)}^{3 p-2}\right),
$$

for a positive constant $C_{1}=C\left(|\Omega|, T, n, p, p_{1}, p_{3}, p_{4}, p_{5}, q_{\min }, q_{\max }, m_{1}, M_{1}\right)$.

Applying $L^{p}$-theory to problem (15) (see [1] and references therein), we get

$$
\begin{aligned}
\|v\|_{W_{p}^{1,2}(Q)} \leq & C_{2}\left(\left\|v_{0}\right\|_{W_{p}^{2-\frac{2}{p}}(\Omega)}+p_{3}\left\|\left(v-v^{3}\right)\right\|_{L^{p}(Q)}\right. \\
& \left.+\|f\|_{L^{p}(Q)}+\|w\|_{W_{p}^{1-\frac{1}{2 p}, 2-\frac{1}{p}}(\Sigma)}\right)
\end{aligned}
$$

for a constant $C_{2}=C\left(|\Omega|, T, n, p, p_{1}, p_{3}, p_{4}\right)>0$.

By Lemma 1.1 in [21] and (24), we get

$$
\left\|v-v^{3}\right\|_{L^{p}(Q)} \leq C_{1}\left(1+\left\|v_{0}\right\|_{L^{3 p-2}(\Omega)}^{\frac{3 p-2}{p}}+\|f\|_{L^{p}(Q)}+\|w\|_{L^{3 p-2}(\Sigma)}^{\frac{3 p-2}{p}}\right)
$$

and then (25) becomes

$$
\begin{aligned}
\|v\|_{W_{p}^{1,2}(Q) \leq} \leq & C_{2}\left(1+\left\|v_{0}\right\|_{W_{\infty}^{2-\frac{2}{p}}(\Omega)}+\left\|v_{0}\right\|_{L^{3 p-2}(\Omega)}^{\frac{3 p-2}{p}}\right. \\
& \left.+\|f\|_{L^{p}(Q)}+\|w\|_{W_{p}^{1-\frac{1}{2 p}, 2-\frac{1}{p}}(\Sigma)}+\|w\|_{L^{3 p-2}\left(\Sigma_{t}\right)}^{\frac{3 p-2}{p}}\right) .
\end{aligned}
$$

The continuous embedding $W_{p}^{1,2}(Q) \subset B=W_{p}^{0,1}(Q) \cap L^{3 p}(Q)$ ensures that

$$
\|v\|_{B} \leq C\|v\|_{W_{p}^{1,2}(Q)}
$$


which, owing to (26), ensures that a constant $\delta>0$ can be found such that the property expressed in (21) is true.

\section{Denoting}

$$
B_{\delta}:=\left\{v \in B:\|v\|_{B}<\delta\right\}
$$

relation (21) implies that

$$
H(v, \lambda) \neq v \quad \forall v \in \partial B_{\delta}, \quad \forall \lambda \in[0,1],
$$

provided that $\delta>0$ is sufficiently large. Furthermore, following the same reasoning as in $[1,4,11,15,19]$, we conclude that problem (6) has a solution $v \in W_{p}^{1,2}(Q)$ (see also [21], p. 195). The estimate (11) results from (26), and the proof of the first part in Theorem 1 is finished.

2.2.2. The Uniqueness of the Solution $v(t, x)$

Now, we prove (13), which implies the uniqueness of the solution of (1) or (5). By hypothesis, $v^{1}, v^{2} \in W_{p}^{1,2}(Q)$ solve problem (1), corresponding to $\left\{f^{1}, w^{1}, v_{0}^{1}\right\}$ and $\left\{f^{2}, w^{2}, v_{0}^{2}\right\}$, respectively. Thus, $v^{1}-v^{2} \in W_{p}^{1,2}(Q)$.

Let us recall that

$$
\begin{aligned}
& a_{i j}\left(t, x, v^{1}, v_{x}^{1}\right)=\frac{\partial}{\partial v_{x_{j}}^{1}} \Phi\left(v_{x}^{1}\right) \Psi\left(v_{x}^{1}\right) v_{x_{i}}^{1} \\
& a_{i j}\left(t, x, v^{2}, v_{x}^{2}\right)=\frac{\partial}{\partial v_{x_{j}}^{2}} \Phi\left(v_{x}^{2}\right) \Psi\left(v_{x}^{2}\right) v_{x_{i}}^{2},
\end{aligned}
$$

$i=1,2$, and (following [1]) we write the increments of $a_{i j}$ in the form

$$
a_{i j}\left(t, x, v^{1}, v_{x}^{1}\right)-a_{i j}\left(t, x, v^{2}, v_{x}^{2}\right)=\int_{0}^{1} \frac{d}{d \lambda} a_{i j}\left(t, x, v^{\lambda}, v_{x}^{\lambda}\right) d \lambda,
$$

where

$$
v^{\lambda}(t, x)=\lambda v^{1}(t, x)+(1-\lambda) v^{2}(t, x) \text { and } v_{x}^{\lambda}(t, x)=\lambda v_{x}^{1}(t, x)+(1-\lambda) v_{x}^{2}(t, x) .
$$

Consequently, we get

$$
\begin{aligned}
& a_{i j}\left(t, x, v^{1}, v_{x}^{1}\right) v_{x_{i} x_{j}}^{1}-a_{i j}\left(t, x, v^{2}, v_{x}^{2}\right) v_{x_{i} x_{j}}^{2} \\
& =a_{i j}\left(t, x, v^{1}, v_{x}^{1}\right) V_{x_{i} x_{j}}+v_{x_{i} x_{j}}^{2}\left[V_{x_{i}} \int_{0}^{1} \frac{\partial}{\partial v_{x_{j}}^{\lambda}} a_{i j}\left(t, x, v^{\lambda}, v_{x}^{\lambda}\right) d \lambda\right. \\
& \left.\quad+V \int_{0}^{1} \frac{\partial}{\partial v^{\lambda}} a_{i j}\left(t, x, v^{\lambda}, v_{x}^{\lambda}\right) d \lambda\right] \\
& \quad+v_{x_{i} x_{j}}^{2}\left[\Phi\left(v_{x}^{1}\right)-\Phi\left(v_{x}^{2}\right)\right],
\end{aligned}
$$

where $V(t, x)=v^{1}(t, x)-v^{2}(t, x)$

Regarding $A\left(t, x, v, v_{x}\right)=\Phi\left(v_{x}\right) \nabla \Psi\left(v_{x}\right) \cdot \nabla v$, we have 


$$
\begin{aligned}
A\left(t, x, v^{1}, v_{x}^{1}\right)-A\left(t, x, v^{2}, v_{x}^{2}\right) \\
\quad=\int_{0}^{1} \frac{d}{d \lambda} A\left(t, x, v^{\lambda}, v_{x}^{\lambda}\right) d \lambda \\
=V_{x_{i}} \int_{0}^{1} \frac{\partial}{\partial v_{x_{j}}^{\lambda}} A\left(t, x, v^{\lambda}, v_{x}^{\lambda}\right) d \lambda+V \int_{0}^{1} \frac{\partial}{\partial v^{\lambda}} A\left(t, x, v^{\lambda}, v_{x}^{\lambda}\right) d \lambda .
\end{aligned}
$$

Now, we subtract Equation $(1)_{1}$ for $v^{2}(t, x)$ from Equation $(1)_{1}$ for $v^{1}(t, x)$, and making use of (27), (28), we obtain the following linear equation

$$
\begin{cases}p_{1} \frac{\partial}{\partial t} V-\hat{a}_{i j}(t, x) V_{x_{i} x_{j}}+\hat{a}_{i}(t, x) V_{x_{i}}+\hat{a}(t, x) V=f^{1}-f^{2} & \text { in } Q \\ \bar{q}(t, x) \frac{\partial}{\partial \mathbf{n}} V+p_{5} V=w^{1}-w^{2} & \text { on } \Sigma \\ V(0, x)=v_{0}^{1}(x)-v_{0}^{2}(x) & \text { on } \Omega,\end{cases}
$$

where

$$
\begin{aligned}
\hat{a}_{i j}(t, x) & =a_{i j}\left(t, x, v^{1}, v_{x}^{1}\right), \\
\hat{a}_{i}(t, x) & =-v_{x_{i} x_{j}}^{2} \int_{0}^{1} \frac{\partial}{\partial v_{x_{j}}^{\lambda}} a_{i, j}\left(t, x, v^{\lambda}, v_{x}^{\lambda}\right) d \lambda+\int_{0}^{1} \frac{\partial}{\partial v_{x_{j}}^{\lambda}} A\left(t, x, v^{\lambda}, v_{x}^{\lambda}\right) d \lambda, \\
\hat{a}(t, x)= & -v_{x_{i} x_{j}}^{2} \int_{0}^{1} \frac{\partial}{\partial v^{\lambda}} a_{i, j}\left(t, x, v^{\lambda}, v_{x}^{\lambda}\right) d \lambda+\int_{0}^{1} \frac{\partial}{\partial v^{\lambda}} A\left(t, x, v^{\lambda}, v_{x}^{\lambda}\right) d \lambda \\
& -p_{2}\left[1-\left(\left(v^{1}\right)^{2}+v^{1} v^{2}+\left(v^{2}\right)^{2}\right)\right] .
\end{aligned}
$$

Due to (9) and the working hypotheses on $v^{1}$ and $v^{2}$, i.e.,

$$
\left\|v^{1}\right\|_{W_{p}^{1,2}(Q)},\left\|v^{2}\right\|_{W_{p}^{1,2}(Q)} \leq M_{4^{\prime}}
$$

the conditions on linear equations are fulfilled and, given this, it follows from (29) that estimate (13) is valid for $V$, which finishes the proof of Theorem 1.

As a consequence, it results the uniqueness for the solution of (5).

Corollary 1. For the same initial conditions, the problem (5) possesses a unique solution $v(t, x) \in$ $W_{p}^{1,2}(Q)$.

Proof. Let $f^{1}=f^{2}=f$ and $w^{1}=w^{2}=w$ in Theorem 1. Then (13) demonstrates the corollary (see [1] and references therein).

Remark 1. The nonlinear operator $H$ in (14) depends on $\lambda \in[0,1]$ and its fixed point for $\lambda=1$ are solutions of (15). 


\section{A Novel Nonlinear Second-Order Anisotropic Reaction-Diffusion Model in Image Segmentation}

The nonlinear parabolic second-order PDE problem (5) can be applied for image denoising, enhancement, restoration and segmentation. Here we consider a particularization of this mathematical model by setting the functions $\Phi\left(v_{x}(t, x)\right)$ and $\Psi\left(v_{x}(t, x)\right)$ as follow

$$
\begin{aligned}
& \Phi:[0, \infty) \rightarrow(0, \infty), \quad \Phi(s)=\frac{\sqrt[3]{\varphi s^{2}+\eta}}{\alpha}, \\
& \Psi:[0, \infty) \rightarrow(0,1], \quad \Psi(s)=\frac{1}{1+\left(\frac{s}{c}\right)^{2}}
\end{aligned}
$$

where $\varphi, \eta, \alpha \in(0,4]$, while the parameter $c$ is the conductance (see [15], p. 177 and [14], p. 633). Therefore, the following PDE scheme with non-homogeneous Cauchy-Neumann boundary conditions is acquired:

$$
\begin{cases}p_{1} \frac{\partial}{\partial t} v(t, x)-\Phi\left(\left\|v_{x}(t, x)\right\|\right) \operatorname{div}\left(\Psi\left(\left\|v_{x}(t, x)\right\|\right) v_{x}(t, x)\right) & \\ =p_{3}\left[v(t, x)-v^{3}(t, x)\right]+p_{4} f(t, x) & \text { in } Q \\ \frac{\partial}{\partial \mathbf{n}} v(t, x)+p_{5} v(t, x)=w(t, x) & \text { on } \Sigma \\ v(0, x)=v_{0}(x) & \text { on } \Omega,\end{cases}
$$

$v_{x}(t, x)=\nabla v(t, x)=\left(v_{x_{1}}(t, x), v_{x_{2}}(t, x)\right)$.

The edge-stopping (diffusivity) function in $(30)_{2}$ is positive, monotonically decreasing and converging to zero (see $[28,30]$ ) thus satisfying the conditions imposed by a proper diffusion. Moreover, it is easy to check that $\Psi$ and $\Phi$ in (30) satisfy Assumptions $I_{1}$ and $\mathrm{I}_{2}$ in Theorem 1 and thus the nonlinear anisotropic reaction-diffusion model (31) is wellposed, as proved in the previous section. Consequently, it admits an unique classical solution $v(t, x) \in W_{p}^{1,2}(Q)$, that represents the evolving image of the observed image $v(0, x)=v_{0}(x)$.

The corresponding nonlocal anisotropic reaction-diffusion model to (31) can be written as follows:

$$
\begin{aligned}
& p_{1} \frac{\partial}{\partial t} v(t, x)=\Phi\left(\left\|v_{x}(t, x)\right\|\right) \Psi\left(\left\|v_{x}(t, x)\right\|\right)\left\{\int_{\Omega} K(x-y)[v(t, y)-v(t, x)] d y\right. \\
& \left.\quad+\int_{\partial \Omega} K\left(x-y_{s}\right)\left[w\left(t, y_{s}\right)-p_{5} v\left(t, y_{s}\right)\right] d y_{s}\right\} \\
& \quad+\Phi\left(\left\|v_{x}(t, x)\right\|\right) \nabla \Psi\left(\left\|v_{x}(t, x)\right\|\right) \cdot v_{x}(t, x)+p_{3}\left[v(t, x)-v^{3}(t, x)\right]+p_{4} f(t, x)
\end{aligned}
$$

with initial condition

$$
v(0, x)=v_{0}(x)
$$

where

- $\quad K: \mathbb{R} \rightarrow \mathbb{R}$ is a real function, symmetric, continuous, nonnegative and it's compactly supported in the unit sphere, such that $\int_{\mathbb{R}} K(z) d z=1$. 
Details on certain interpretations of the terms $K(x-y), \int_{\Omega} K(x-y) v(t, y) d y$ and $-v(t, x) \int_{\Omega} K(x-y) d y$ in the mathematical model (32), can be found in the works of P. W.

Bates, S. Brown and J. Han [3] and J. Rubinstein and P. Sternberg [27] and references therein. The solution behavior for the nonlocal model (32) on rescaling the kernel $K$ considering $K(z)=\frac{1}{\varepsilon^{3}} K\left(\frac{z}{\varepsilon}\right)$ are studied in [33] and for the numerical solutions we refer to [3,40] and references therein.

In what follows, we will approximate the solution $v(t, x)$ in (31) and (32) using the finite-difference method (of second-order in time, see (36)).

\subsection{Numerical Approximation}

In this subsection we propose two numerical schemes (see (47) and (48)) to approximate the solution of the novel nonlinear reaction-diffusion model (31), (32), based on the finite difference method (see also $[3,4,7,9,16,23,28,40,41])$. By using a grid of space size $h$, one quantizes the space coordinates $x=(x 1, x 2)$ as:

$$
x 1_{i}=i h, x 2_{j}=j h, \quad \text { for all } i=1,2 \ldots, I, j=1,2, \ldots, J,
$$

where $[I h \times J h]$ represents the dimension of the support image.

We consider a positive value $T$ as the time interval upper limit and $M$ the number of nodes which are dividing the time interval $[0, T]$, then we can set

$$
t_{m}=(m-1) \varepsilon, \quad m=1,2, \ldots, M, \quad \varepsilon=T /(M-1) .
$$

We also denote by $v_{i, j}^{m}$ the approximating values in $\left(t_{m}, x 1_{i}, x 2_{j}\right)$ for the unknown function $v(t, x)$ used in (31) (or (32)), i.e.,

$$
v_{i, j}^{m}=v\left(t_{m}, x 1_{i}, x 2_{j}\right), \quad m=1,2, \ldots, M, \quad i=1,2 \ldots, I, \quad j=1,2, \ldots, J,
$$

or, for later use

$$
v^{m}=\left(v_{1,1}^{m}, v_{2,1}^{m}, \ldots, v_{I h, J h}^{m}\right)^{T} \quad m=1,2, \ldots, M
$$

From the initial condition (33), we have

$$
v(0, x) \approx v^{1}=v\left(t_{1}, x 1_{i}, x 2_{j}\right)=v_{0}\left(x 1_{i}, x 2_{j}\right), \quad i=1,2 \ldots, I, \quad j=1,2, \ldots, J .
$$

To approximate $\frac{\partial}{\partial t} v(t, x)$, we employ a second-order scheme (see [16,41] and references therein):

$$
\begin{aligned}
\frac{\partial}{\partial t} v\left(t_{m+1}, x 1_{i}, x 2_{j}\right) & \approx \frac{3 v_{i, j}^{m+1}-4 v_{i, j}^{m}+v_{i, j}^{m-1}}{2 \varepsilon}, \\
& m=1,2, \ldots, M-1, \quad i=1,2 \ldots, I, \quad j=1,2, \ldots, J .
\end{aligned}
$$

We write Equation in (32) as:

$$
p_{1} \frac{\partial}{\partial t} v(t, x)+p_{3}\left[v^{3}(t, x)-v(t, x)\right]=N l D\left(t, x, v, v_{x}\right)+R\left(t, x, v, v_{x}\right)+p_{4} f(t, x)
$$

where we denote the nonlocal diffusion term by: 


$$
\begin{aligned}
N l D\left(t, x, v, v_{x}\right)= & \Phi\left(\left\|v_{x}(t, x)\right\|\right) \Psi\left(\left\|v_{x}(t, x)\right\|\right)\left\{\int_{\Omega} K(x-y)[v(t, y)-v(t, x)] d y\right. \\
& \left.+\int_{\partial \Omega} K\left(x-y_{s}\right)\left[w\left(t, y_{s}\right)-p_{5} v\left(t, y_{s}\right)\right] d y_{s}\right\}
\end{aligned}
$$

and the reaction term by:

$$
R\left(t, x, v, v_{x}\right)=\Phi\left(\left\|v_{x}(t, x)\right\|\right) \nabla \Psi\left(\left\|v_{x}(t, x)\right\|\right) \cdot v_{x}(t, x) .
$$

The left-side term in (37) is approximated by

$$
p_{1} \frac{3 v_{i, j}^{m+1}-4 v_{i, j}^{m}+v_{i, j}^{m-1}}{2 \varepsilon}+p_{3}\left[\left(v_{i, j}^{m}\right)^{3}-v_{i, j}^{m}\right]
$$

and the right side terms are discretized using central differences (see [16] and references therein).

$$
\begin{aligned}
& \text { We also denote } \Phi_{i, j}=\Phi\left(\left\|\nabla v_{i, j}\right\|\right) \text { and } \Psi_{i, j}=\Psi\left(\left\|\nabla v_{i, j}\right\|\right) \text {, where } \\
& \left\|\nabla v_{i, j}\right\|=\left\|v_{x}\left(t, x_{i, j}\right)\right\| \approx \sqrt{\left(\frac{v_{i+1, j}^{m}-v_{i-1, j}^{m}}{2 h}\right)^{2}+\left(\frac{v_{i, j+1}^{m}-v_{i, j-1}^{m}}{2 h}\right)^{2}},
\end{aligned}
$$

for all $i=2 \ldots, I-1, j=2, \ldots, J-1$. To complete the discretization schema we need to approximate $N l D\left(t, x, v, v_{x}\right)$ and $R\left(t, x, v, v_{x}\right)$ terms as follows:

$$
\begin{aligned}
& N l D^{m+1}\left(t_{m}, x_{i, j}, v_{i, j}^{m}, v_{x}\left(t_{m}, x_{i, j}\right)\right)= \\
& \Phi_{i, j}^{m} \Psi_{i, j}^{m}\left\{\int_{\Omega} K\left(x_{i, j}-y\right)\left[v\left(t_{m}, y\right)-v\left(t_{m}, x_{i, j}\right)\right] d y\right. \\
& \left.\quad+\int_{\partial \Omega} K\left(x_{i, j}-y_{s}\right)\left[w\left(t_{m}, y_{s}\right)-p_{5} v\left(t_{m}, x_{i, j}\right)\right] d y_{s}\right\}
\end{aligned}
$$

Continuing the discretization by using the Riemann sums to approximate the integral terms, we have: 


$$
\begin{aligned}
& \int_{\Omega} K\left(x_{i, j}-y\right) {\left[v^{m}\left(t_{m}, y\right)-v^{m}\left(t_{m}, x_{i, j}\right)\right] d y=} \\
& h^{2}\left\{\sum_{d_{1}=2 d_{2}=2}^{I-1} \sum^{J-1} K\left(x_{i, j}-y_{d_{1}, d_{2}}\right)\left(v_{d_{1}, d_{2}}^{m}-v_{i, j}^{m}\right)\right. \\
&+\frac{1}{2} \sum_{d_{1}=2}^{I-1}\left[K\left(x_{i, j}-y_{d_{1}, 1}\right)\left(v_{d_{1}, 1}^{m}-v_{i, j}^{m}\right)+K\left(x_{i, j}-y_{d_{1}, J}\right)\left(v_{d_{1}, J}^{m}-v_{i, j}^{m}\right)\right] \\
&+\frac{1}{2} \sum_{d_{2}=2}^{J-1}\left[K\left(x_{i, j}-y_{1, d_{2}}\right)\left(v_{1, d_{2}}^{m}-v_{i, j}^{m}\right)+K\left(x_{i, j}-y_{I, d_{2}}\right)\left(v_{I, d_{2}}^{m}-v_{i, j}^{m}\right)\right] \\
&+\frac{1}{4}\left[K\left(x_{i, j}-y_{1,1}\right)\left(v_{1,1}^{m}-v_{i, j}^{m}\right)+K\left(x_{i, j}-y_{I, 1}\right)\left(v_{I, 1}^{m}-v_{i, j}^{m}\right)\right. \\
&\left.\left.\quad+K\left(x_{i, j}-y_{1, J}\right)\left(v_{1, J}^{m}-v_{i, j}^{m}\right)+K\left(x_{i, j}-y_{I, J}\right)\left(v_{I, J}^{m}-v_{i, j}^{m}\right)\right]\right\}
\end{aligned}
$$

For the second integral on $\partial \Omega$, we have:

$$
\begin{aligned}
& \int_{\partial \Omega} K\left(x_{i, j}-y_{s}\right)\left[w\left(t_{m}, y_{s}\right)-p_{5} v\left(t_{m}, x_{i, j}\right)\right] d y_{s} \\
& =h\left\{\sum_{d_{1}=2}^{I-1}\left[K\left(x_{i, j}-y_{d_{1}, 1}\right)\left(w_{d_{1}, 1}^{m}-p_{5} v_{i, j}^{m}\right)+K\left(x_{i, j}-y_{d_{1}, J}\right)\left(w_{d_{1, J}}^{m}-p_{5} v_{i, j}^{m}\right)\right]\right. \\
& \quad+\sum_{d_{2}=2}^{J-1}\left[K\left(x_{i, j}-y_{1, d_{2}}\right)\left(w_{1, d_{2}}^{m}-p_{5} v_{i, j}^{m}\right)+K\left(x_{i, j}-y_{I, d_{2}}\right)\left(w_{I, d_{2}}^{m}-p_{5} v_{i, j}^{m}\right)\right] \\
& \quad+K\left(x_{i, j}-y_{1,1}\right)\left(w_{1,1}^{m}-p_{5} v_{i, j}^{m}\right)+K\left(x_{i, j}-y_{I, 1}\right)\left(w_{I, 1}^{m}-p_{5} v_{i, j}^{m}\right) \\
& \left.\quad+K\left(x_{i, j}-y_{1, J}\right)\left(w_{1, J}^{m}-p_{5} v_{i, j}^{m}\right)+K\left(x_{i, j}-y_{I, J}\right)\left(w_{I, J}^{m}-p_{5} v_{i, j}^{m}\right)\right\}
\end{aligned}
$$

For the reaction term discretization,

$$
R^{m}\left(t_{m}, x_{i, j}, v_{i, j}^{m}, v_{x}\left(t_{m}, x_{i, j}\right)\right)=\Phi_{i, j}^{m} \nabla \Psi\left(\left\|v_{x}\left(t_{m}, x_{i, j}\right)\right\|\right) \cdot v_{x}\left(t_{m}, x_{i, j}\right)
$$

we use the following scalar product approximation:

$$
\begin{aligned}
& \nabla \Psi\left(\left\|v_{x}(., x 1, x 2)\right\|\right) \cdot v_{x}(., x 1, x 2) \\
& =\left(\frac{\partial}{\partial x 1} \Psi\left(\sqrt{\left(\frac{\partial v}{\partial x 1}\right)^{2}+\left(\frac{\partial v}{\partial x 2}\right)^{2}}\right), \frac{\partial}{\partial x 2} \Psi\left(\sqrt{\left(\frac{\partial v}{\partial x 1}\right)^{2}+\left(\frac{\partial v}{\partial x 2}\right)^{2}}\right)\right) \cdot\left(\frac{\partial v}{\partial x 1}, \frac{\partial v}{\partial x 2}\right)
\end{aligned}
$$

which leads to

$$
\begin{aligned}
& \nabla \Psi\left(\left\|v_{x}(., x 1, x 2)\right\|\right) \cdot v_{x}(., x 1, x 2) \\
& =\frac{\partial \Psi}{\partial s}\left(\left\|v_{x}(., x 1, x 2)\right\|\right) \frac{\left(\frac{\partial v}{\partial x 1}\right)^{2} \frac{\partial^{2} v}{\partial x 1^{2}}+\frac{\partial v}{\partial x 1} \frac{\partial v}{\partial x 2} \frac{\partial^{2} v}{\partial x 1 \partial x 2}+\left(\frac{\partial v}{\partial x 2}\right)^{2} \frac{\partial^{2} v}{\partial x 2^{2}}+\frac{\partial v}{\partial x 1} \frac{\partial v}{\partial x 2} \frac{\partial^{2} v}{\partial x 1 \partial x 2}}{\sqrt{\left(\frac{\partial v}{\partial x 1}\right)^{2}+\left(\frac{\partial v}{\partial x 2}\right)^{2}}}
\end{aligned}
$$


Further, since the second-order derivatives do not vary too much, we can use

$$
\frac{\partial^{2} v}{\partial x 1^{2}} \approx \frac{\partial^{2} v}{\partial x 1 \partial x 2} \quad \frac{\partial^{2} v}{\partial x 2^{2}} \approx \frac{\partial^{2} v}{\partial x 1 \partial x 2}
$$

to approximate

$$
\begin{aligned}
& \nabla \Psi\left(\left\|v_{x}(., x 1, x 2)\right\|\right) \cdot v_{x}(., x 1, x 2) \\
& \approx \frac{\partial \Psi}{\partial s}(s) \frac{\frac{\partial^{2} v}{\partial x 1 \partial x 2}\left(\frac{\partial v}{\partial x 1}+\frac{\partial v}{\partial x 2}\right)^{2}}{\sqrt{\left(\frac{\partial v}{\partial x 1}\right)^{2}+\left(\frac{\partial v}{\partial x 2}\right)^{2}}} \approx \Psi^{\prime}\left(\sqrt{v_{x 1}^{2}+v_{x 2}^{2}}\right) v_{x 1 x 2}\left(v_{x 1}+v_{x 2}\right),
\end{aligned}
$$

where $v_{x 1}=\partial v / \partial x 1, v_{x 2}=\partial v / \partial x 2$ and $v_{x 1 x 2}=\partial^{2} v / \partial x 1 \partial x 2$ are discretized by applying the finite difference method (see $[15,28]$ ).

To conclude we obtain the following explicit numerical approximation for reaction term:

$$
\begin{aligned}
& R^{m}\left(t_{m}, x_{i, j}, v_{i, j}^{m}, v_{x}\left(t_{m}, x_{i, j}\right)\right)=\Phi_{i, j} \Psi^{\prime}\left(\sqrt{\frac{\left(v_{i+1, j}^{m}-v_{i-1, j}^{m}\right)^{2}}{4 h^{2}}+\frac{\left(v_{i, j+1}^{m}-v_{i, j-1}^{m}\right)^{2}}{4 h^{2}}}\right) \\
& \cdot \frac{\left(v_{i+1, j+1}^{m}-v_{i+1, j-1}^{m}-v_{i-1, j+1}^{m}+v_{i-1, j-1}^{m}\right)\left(v_{i+1, j}^{m}-v_{i-1, j}^{m}+v_{i, j+1}^{m}-v_{i, j-1}^{m}\right)}{8 h^{3}}
\end{aligned}
$$

and thus we get the following explicit numerical approximation scheme for (32):

$$
\frac{3 p_{1}}{2 \varepsilon} v_{i, j}^{m+1}=\left(\frac{2 p_{1}}{\varepsilon}+p_{3}\right) v_{i, j}^{m}-p_{3}\left(v_{i, j}^{m}\right)^{3}-\frac{p_{1}}{2 \varepsilon} v_{i, j}^{m-1}+N I D_{i, j}^{m}+R_{i, j}^{m}+p_{4} f_{i, j}^{m} .
$$

In a similar manner one obtains the following explicit numerical approximation scheme for (31):

$$
\begin{aligned}
\frac{3 p_{1}}{2 \varepsilon} v_{i, j}^{m+1}= & \left(\frac{2 p_{1}}{\varepsilon}+p_{3}\right) v_{i, j}^{m}-p_{3}\left(v_{i, j}^{m}\right)^{3}-\frac{p_{1}}{2 \varepsilon} v_{i, j}^{m-1} \\
+ & +\Psi_{i, j}\left[K_{i+\frac{1}{2}, j}\left(v_{i+1, j}^{m}-v_{i, j}^{m}\right)-K_{i-\frac{1}{2}, j}\left(v_{i, j}^{m}-v_{i-1, j}^{m}\right)\right. \\
& \left.\quad+K_{i, j+\frac{1}{2}}\left(v_{i, j+1}^{m}-v_{i, j}^{m}\right)-K_{i, j-\frac{1}{2}}\left(v_{i, j}^{m}-v_{i, j-1}^{m}\right)\right] .
\end{aligned}
$$

\subsection{Experimental Results}

The iterative numerical approximation scheme provided by (47) was successfully applied in our image segmentation experiments, for each $m=1,2, \ldots, M-1$, starting with $v^{1}=v_{0}(x)$ (see (33)), which represents the $[I h \times J h]$ image to be segmented.

The explicit numerical approximation scheme developed in (47) is consistent to the nonlinear second-order anisotropic reaction-diffusion model given by (32).

In summary, the computations follow the procedure in Algorithm 1. For our tests, we used the following parameter values: $h=1, \epsilon=0.1, p_{1}=0.6, p_{3}=50, p_{4}=$ $1, p_{5}=0.3, \alpha=1, \eta=3$ and $\phi=1$.

Some image segmentation results provided by our proposed model are displayed in Figures 1-4. All the results presented in this section are compared to standard K-means image segmentation model with two clusters [24] and the Chan-Vese image segmentation model presented in [5].

Our model successfully extracts the objects after up to three iterations. One may see multiple objects as well as objects with boundary concavities and blurry boundaries are accurately extracted from the background. 


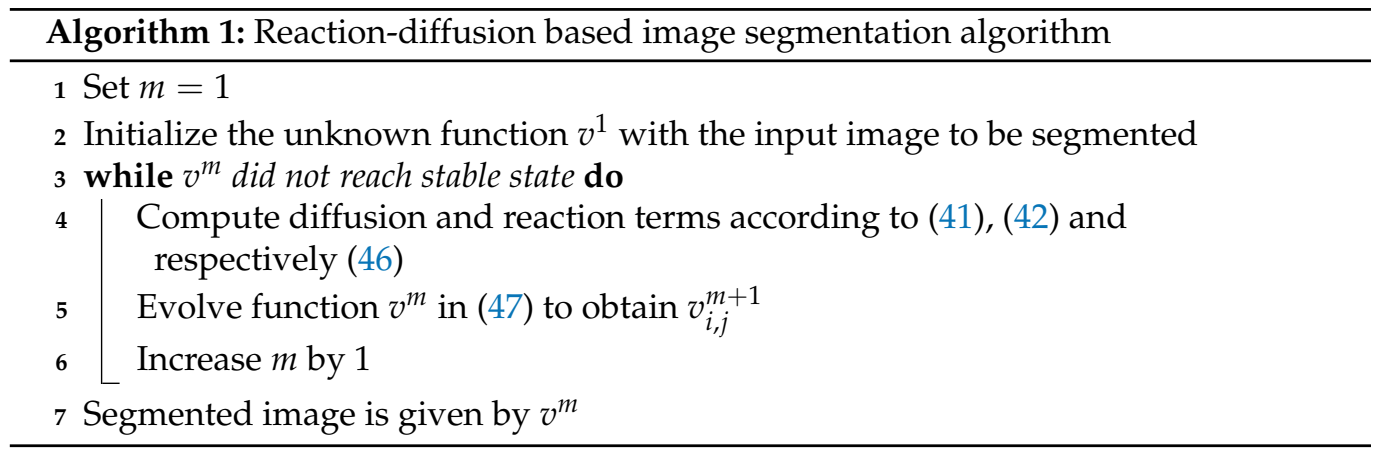

Figure 1 shows the segmentation results of our model for a brain CT scan image. The results are satisfactory even after only one iteration. We also see the model reaching stability after two iterations in this case. Compared to K-means segmentation results, we observe the extracted objects edges (brain tissue and cranium bone) are better delimited from the background. Compared to Chan-Vese segmentation results, our model produces more accurate results too. In this example, Chan-Vese model seems to not follow the real object boundaries, especially at the border between cranium bone and brain tissue.

Figure 2 shows the segmentation comparison between three cases: first the input image is segmented 'as is', second the input image is contaminated with noise before segmentation and third we double the noise added to the input image. For all three cases, we can also see the results of applying K-means and Chan-Vese segmentation. We see our model successfully removes most part of the noise in Figure $2 \mathrm{~h}, 1$ while still preserving a good approximation for the edges on the leaf object (better than both K-means and Chan-Vese).

In Figure 3, we see the segmentation results for a blurry boundary object as galaxy boundaries are slowly fading. Even after one iteration, our segmentation is superior to K-means and Chan-Vese as the real galaxy boundaries are correctly identified in Figure 3d.

Figure 4 (virus microscopy) brings together noise, blur and irregular boundaries. Again, after two iterations, the model successfully identifies all objects of interest and the results, starting with the first iteration, are better than the compared K-means method. The Chan-Vese segmentation does not separate the virus blobs successfully, although it provides a good outer boundary approximation.

Regarding time complexity, due to the integral formulation of NlD term in (41) and (42), the proposed algorithm is slower than the compared K-means or Chan-Vese counterparts. To obtain better performance results, regarding running time, we had to implement the program on parallel architectures such as CUDA [42]. Table 1 shows the time taken by a CUDA implementation for different input image sizes (total number of pixels being $I * J$ ).

Using the local scheme in (48), we obtained promising results for image restoration tasks. Future work will show if we can succeed in mixing the local and nonlocal models for better noise removal before applying segmentation tasks.

Table 1. Running durations for the reaction-diffusion algorithm implemented on CUDA. The durations are for only one $v^{m}$ iteration.

\begin{tabular}{cccc}
\hline $\begin{array}{c}\text { Input Area Size } \\
\text { (Pixels) }\end{array}$ & 65,536 & 262,144 & $1,048,576$ \\
\hline $\begin{array}{c}\text { Time Taken } \\
\text { (Seconds) }\end{array}$ & 0.3 & 2.0 & 30.0 \\
\hline
\end{tabular}



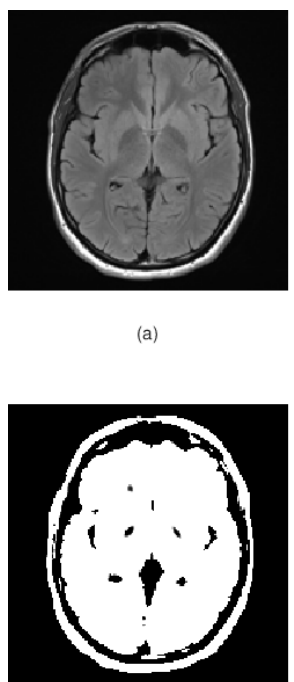

(d)
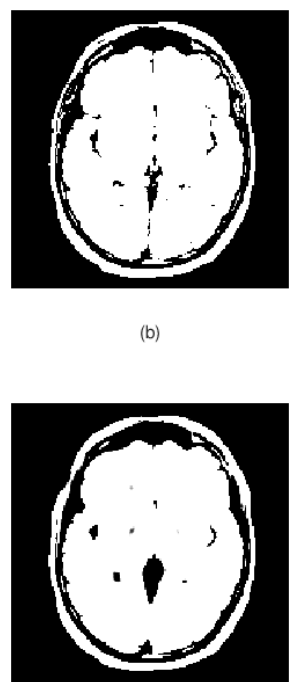

(e)
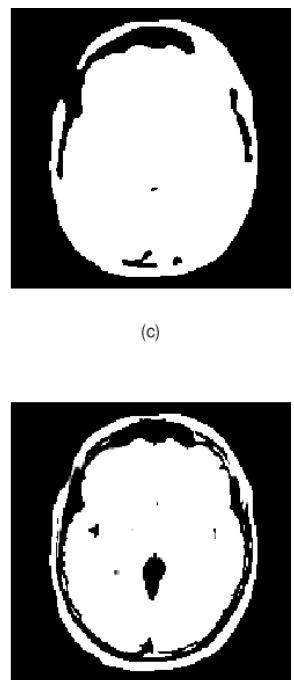

(f)

Figure 1. (a) Original input image to be segmented, (b) K-means segmentation results, (c) Chan-Vese segmentation results; and (d-f) our model segmentation results after 1-3 iterations, respectively.

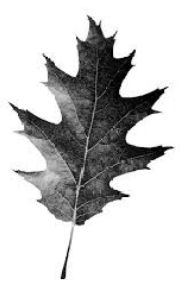

(a)

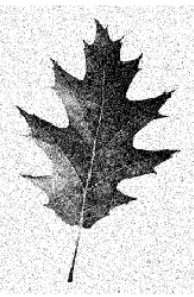

(e)

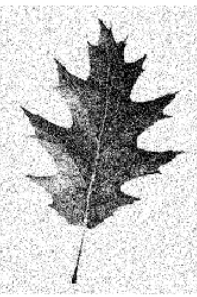

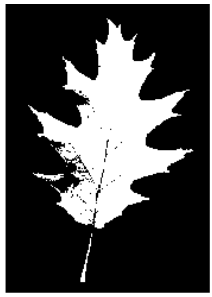

(b)
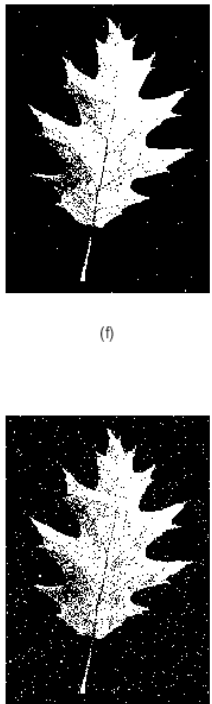

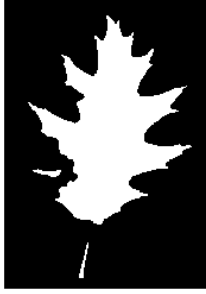

(c)

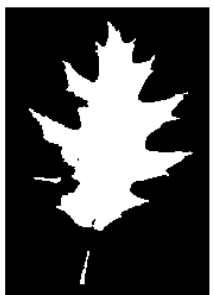

(g)

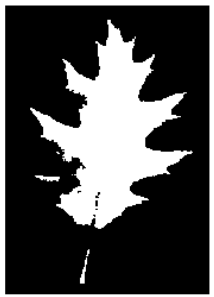

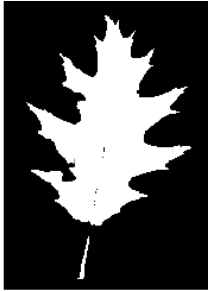
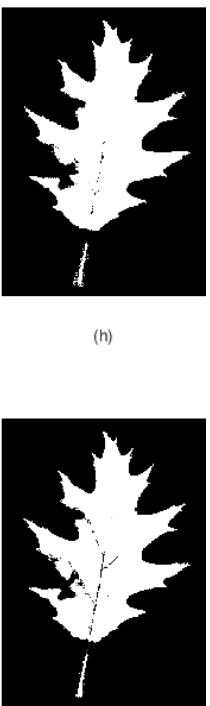

Figure 2. (a) Original input image to be segmented; (b) K-means segmentation results; (c) ChanVese segmentation results; (d) Our model segmentation results after 2 iterations; (e) Input image to be segmented with Gaussian noise added; (f) K-means segmentation results for noisy input in (e); (g) Chan-Vese segmentation results for noisy input in (e); (h) Our model segmentation results for noisy input in (e) after 2 iterations; (i) Input image to be segmented with more noise added; (j) K-means segmentation results for image in (g); (k) Chan-Vese segmentation results for noisy input in (g); and (1) Our model segmentation results for noisy image in (g). 


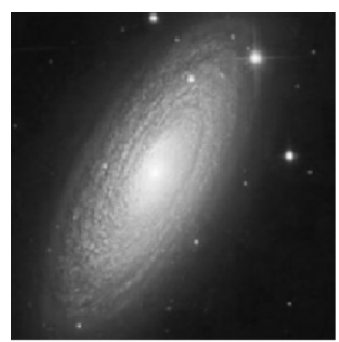

(a)

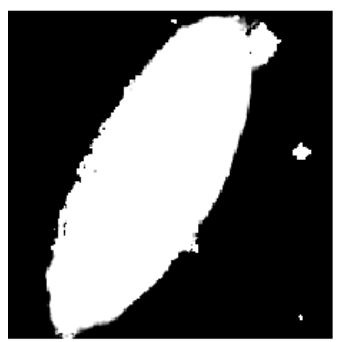

(d)

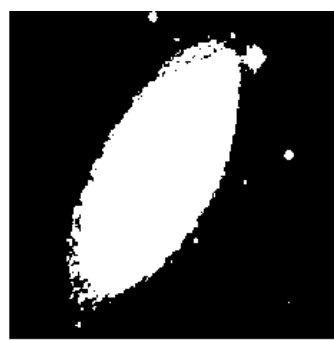

(b)

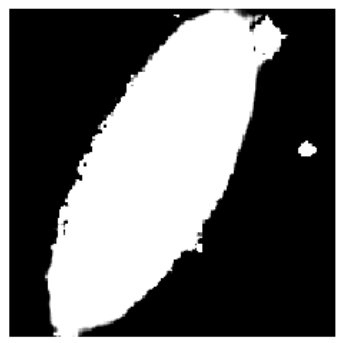

(e)

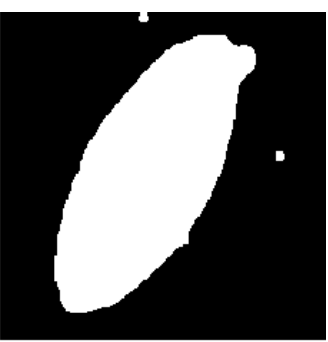

(c)

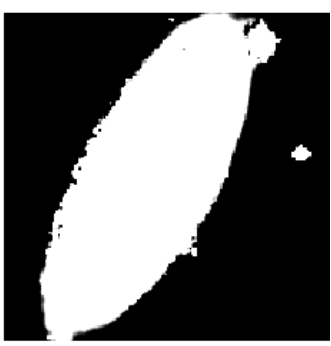

(f)

Figure 3. (a) Input image to be segmented; (b) K-means segmentation results; (c) Chan-Vese segmentation results; and (d-f) Our model segmentation results after 1-3 iterations. respectively.

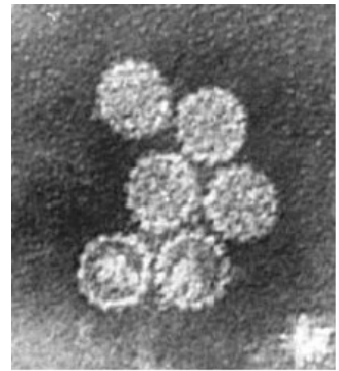

(a)

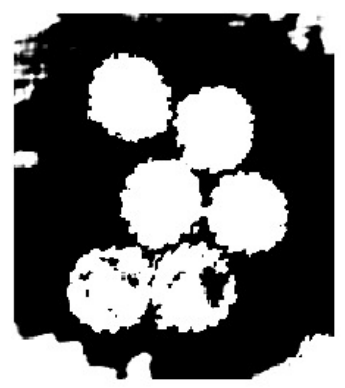

(d)

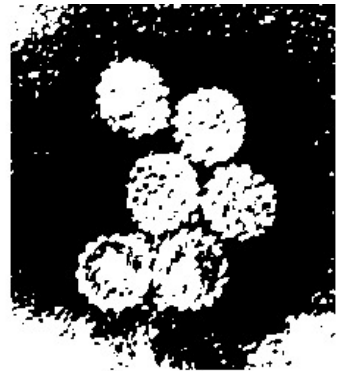

(b)

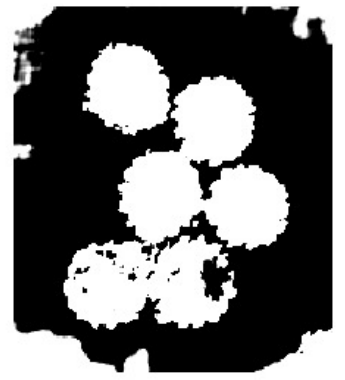

(e)

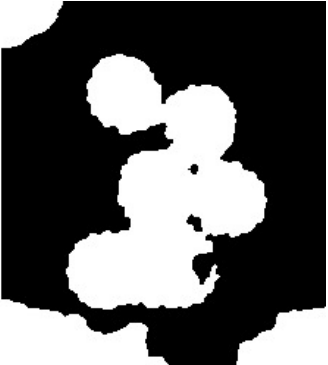

(c)

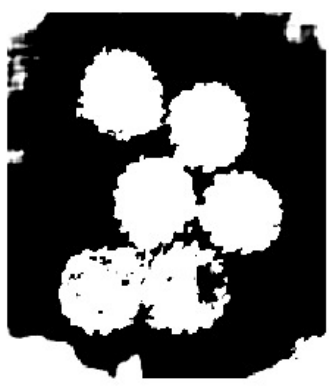

(f)

Figure 4. (a) Original input image to be segmented; (b) K-means segmentation results; (c) Chan-Vese segmentation results; (d-f) Our model segmentation results after 1-3 iterations. respectively.

\section{Conclusions}

The starting point in the elaboration of the present work is the paper by Miranville, A. and Moroşanu, C. [1], which is a major challenge for both theory and applications, focused on finding concrete cases of functions for the general case $\Phi\left(t, x, v(t, x), v_{x}(t, x)\right)$ and $\Psi\left(t, x, v(t, x), v_{x}(t, x)\right)$ introduced in [1]. In this respect, a rigorous mathematical investigation is performed to analyze the well-posedness of the nonlinear anisotropic reaction-diffusion model (1) (in particular, (31)). The Leray-Schauder principle is applied 
to prove the existence and uniqueness of a unique classical solution $v(t, x) \in W_{p}^{1,2}(Q)$, while the $L^{p}$ theory is used to derive the regularity properties for the solutions, considering that the initial data and the boundary constraints are compatible with the regularity and compatibility conditions (see (3)). In addition, the a priori estimates are made in $L^{p}(Q)$, which means the approximation for unknown functions $v(t, x)$ are more precise (see [1,11-13,15,19-21,35]).

Using the finite-difference method (of second-order in time), two numerical schemes are constructed see (47) and (48) to approximate the solution $v(t, x)$ of the new mathematical model. Numerical experiments show the model can be successfully applied to image segmentation tasks. We tested on images with multiple objects as well as objects with complex concavities or blurry boundaries and proved our model can accurately extract them, most of the time showing better results than the compared K-means model.

Summarizing, the main contributions in the present work are as follows:

- We use novel techniques, such as Leray-Schauder principle, a priori estimates, $L^{p}$ theory, to elaborate a rigorous qualitative study of the nonlocal and nonlinear secondorder anisotropic reaction-diffusion parabolic problem, endowed with a nonlinearity of cubic type as well as non-homogeneous Cauchy-Neumann boundary conditions, expressed by (1) and (31). We note that, due to the presence of the nonlinear coefficient $\Phi\left(\| v_{x}(t, x)\right) \|$ (see (30)), the proposed second-order nonlinear reaction-diffusion scheme (31) represents a non-variational PDE model. Therefore, it cannot be obtained from a minimization of any energy cost functional, thus this scheme is not a variational PDE model.

- Two two numerical schemes (47) and (48) are constructed to approximate the solution of the mathematical models (31) and (32) (local and nonlocal case).

Regarding the second theme, we aim to improve the scheme in (47) and (48), as part of our future research on the topic, by introducing new edge-stopping functions (see [28]) and by taking advantage of non-local image information which will allow us to apply the model to images with inhomogeneity (see [33] and reference therein).

The qualitative results obtained in this current work can be used in quantitative studies of the mathematical models in (1) or (5) as well as in the study of optimal control problems involving such nonlinear problems. We look forward to exploiting all these in our future works.

Author Contributions: Conceptualization, C.M.; Formal analysis, S.P.; Project administration, C.M.; Software, C.M. and S.P.; Supervision, C.M.; Validation, S.P.; Writing-original draft, C.M. and S.P. Both authors have read and agreed to the published version of the manuscript.

Funding: This research received no external funding.

Institutional Review Board Statement: Not applicable.

Informed Consent Statement: Not applicable.

Data Availability Statement: Data sharing not applicable.

Conflicts of Interest: The authors declare no conflict of interest.

\section{References}

1. Miranville, A.; Moroşanu, C. A Qualitative Analysis of a Nonlinear Second-Order Anisotropic Diffusion Problem with Nonhomogeneous Cauchy-Stefan-Boltzmann Boundary Conditions. Appl. Math. Optim. 2019. [CrossRef]

2. Allen, S.M.; Cahn, J.W. A microscopic theory for antiphase boundary motion and its application to antiphase domain coarsening. Acta Metall. 1979, 27, 1085-1095. [CrossRef]

3. Bates, P.W.; Brown, S.; Han, J. Numerical analysis for a nonlocal Allen-Cahn equation. Int. J. Numer. Anal. Model. $2009,6,33-49$.

4. Bogoya, M.; Gómez, J. On a nonlocal diffusion model with Neumann boundary conditions. Nonlinear Anal. 2012, 75, 3198-3209. [CrossRef]

5. Chan, T.F.; Vese, L.A. Active contours without edges. IEEE Trans. Image Process. 2001, 10, 266-277. [CrossRef]

6. Caginalp, G.; Lin, J.-T. A numerical analysis of an anisotropic phase field model. IMA J. Appl. Math. 1987, 39, 51-66. [CrossRef] 
7. Hundsdorfer, W.; Verwer, J. Numerical Solution of Time-Dependent Advection-Diffusion-Reaction Equations; Springer Series in Computational Mathematics; Springer: Berlin/Heidelberg, Germany, 2003; Volume 33.

8. de Masi, A.; Orlandi, E.; Presutti, E.; Triolo, L. Stability of the interface in a model of phase separation. Proc. R. Soc. Edin. A 1994, 124, 1013-1022. [CrossRef]

9. Moroşanu, C. Approximation of the phase-field transition system via fractional steps method. Numer. Funct. Anal. Optimiz. 1997, 18, 623-648. [CrossRef]

10. Moroşanu, C. Cubic spline method and fractional steps schemes to approximate the phase-field system with non-homogeneous Cauchy-Neumann boundary conditions. ROMAI J. 2012, 8, 73-91.

11. Moroşanu, C. Analysis and Optimal Control of Phase-Field Transition System: Fractional Steps Methods; Bentham Science Publishers: Sharjah, UAE, 2012. [CrossRef]

12. Moroşanu, C. Well-posedness for a phase-field transition system endowed with a polynomial nonlinearity and a general class of nonlinear dynamic boundary conditions. J. Fixed Point Theory Appl. 2016, 18, 225-250. [CrossRef]

13. Moroşanu, C. Qualitative and quantitative analysis for a nonlinear reaction-diffusion equation. ROMAI J. 2016, 12, 85-113 Available online: https:/ /rj.romai.ro/arhiva/2016/2/Morosanu.pdf (accessed on 13 December 2020).

14. Moroşanu, C.; Croitoru, A. Analysis of an iterative scheme of fractional steps type associated to the phase-field equation endowed with a general nonlinearity and Cauchy-Neumann boundary conditions. J. Math. Anal. Appl. 2015, 425, 1225-1239. [CrossRef]

15. Barbu, T.; Miranville, A.; Moroşanu, C. A qualitative analysis and numerical simulations of a nonlinear second-order anisotropic diffusion problem with non-homogeneous Cauchy-Neumann boundary conditions. Appl. Math. Comput. 2019, 350, 170-180. [CrossRef]

16. Moroşanu, C.; Pavăl, S.; Trenchea, C. Analysis of stability and errors of three methods associated to the nonlinear reaction-diffusion equation supplied with homogeneous Neumann boundary conditions. J. Appl. Anal. Comput. 2017, 7, 1-19. [CrossRef]

17. Ovono, A.A. Numerical approximation of the phase-field transition system with non-homogeneous Cauchy-Neumann boundary conditions in both unknown functions via fractional steps methods. JAAC 2013, 3, 377-397. [CrossRef]

18. Ignat, L.I.; Rossi, J.D. A nonlocal convection-diffusion equation. J. Funct. Anal. 2007, 251, 399-437. [CrossRef]

19. Cârjă, O.; Miranville, A.; Moroşanu, C. On the existence, uniqueness and regularity of solutions to the phase-field system with a general regular potential and a general class of nonlinear and non-homogeneous boundary conditions. Nonlinear Anal. TMA 2015, 113, 190-208. [CrossRef]

20. Gavriluţ, A.; Moroşanu, C. Well-Posedness for a Nonlinear Reaction-Diffusion Equation Endowed with Nonhomogeneous Cauchy-Neumann Boundary Conditions and Degenerate Mobility. ROMAI J. 2018, 14, 129-141.

21. Moroşanu, C.; Motreanu, D. The phase field system with a general nonlinearity. Int. J. Differ. Equ. Appl. 2000, 1, 187-204.

22. Gonzalez, R.C.; Woods, R.E.; Eddins, S.L. Digital Image Processing Using Matlab, 2nd ed.; Prentice-Hall: Upper Saddle River, NJ, USA, 2010.

23. Jeong, D.; Lee, S.; Lee, D.; Shin, J.; Kim, J. Comparison study of numerical methods for solving the Allen-Cahn equation. Comput. Mater. Sci. 2016, 111, 131-136. [CrossRef]

24. Kanungo, T.; Mount, D.M.; Netanyahu, N.S.; Piatko, C.D.; Silverman, R.; Wu, A.Y. An efficient k-means clustering algorithm: Analysis and implementation. IEEE Trans. Pattern Anal. Mach. Intell. 2002, 24, 881-892. [CrossRef]

25. Lee, D.; Lee, S. Image Segmentation Based on Modified Fractional Allen-Cahn Equation. Math. Probl. Eng. 2019. [CrossRef]

26. Lie, J.; Lysaker, M.; Tai, X.C. A variant of the level set method and applications to image segmentation. Math. Comput. 2006, 75, 1155-1174. [CrossRef]

27. Rubinstein, J.; Sternberg, P. Nonlocal reaction-diffusion equations and nucleation. IMA J. Appl. Math. 1992, 48, 249-264. [CrossRef]

28. Perona, P.; Malik, J. Scale-space and edge detection using anisotropic diffusion. In Proceedings of the IEEE Computer Society Workshop on Computer Vision, San Juan, PR, USA, 17-19 June 1997; pp. 16-22. [CrossRef]

29. Taylor, J.E.; Cahn, J.W. Diffuse interfaces with sharp corners and facets: Phase-field models with strongly anisotropic surfaces. Physics D 1998, 112, 381-411. [CrossRef]

30. Weickert, J. Anisotropic Diffusion in Image Processing. In European Consortium for Mathematics in Industry; B. G. Teubner: Stuttgart, Germany, 1998.

31. Hu, Y.; Jacob, M. Higher degree total variation (HDTV) regularization for image recovery. IEEE Trans. Image Process. 2012, 21, 2559-2571. [CrossRef]

32. Benes, M.; Chalupecky, V.; Mikula, K. Geometrical image segmentation by the Allen-Cahn equation. Appl. Numer. Math. 2004, 51, 187-205. [CrossRef]

33. Bresson, X.; Chan, T. Non-Local Unsupervised Variational Image Segmentation Models; Technical Report; UCLA CAM: Los Angeles, CA, USA, 2008; pp. 8-67.

34. Cortazar, C.; Elgueta, M.; Rossi, J.D.; Wolanski, N. Boundary fluxes for nonlocal diffusion. J. Differ. Equ. 2007, 234, 360-390. [CrossRef]

35. Siddiqi, K.; Lauzière, Y.B.; Tannenbaum, A.; Zucker, S.W. Area and length minimizing flows for shape segmentation. IEEE Trans. Image Process. 1998, 7, 433-443. [CrossRef]

36. Tai, X.C.; Christiansen, O.; Lin, P.; Skjælaaen, I. Image segmentation using some piecewise constant level set methods with MBO type of projection. Int. J. Comput. Vis. 2007, 73, 61-76. [CrossRef] 
37. Vijayakrishna, R.; Kumar, B.V.R.; Halim, A. A PDE Based Image Segmentation Using Fourier Spectral Method. Differ. Equ. Dyn. Syst. 2018. [CrossRef]

38. Gilboa, G.; Osher, S. Nonlocal Linear Image Regularization and Supervised Segmentation. Multiscale Model. Simul. 2007, 6, 595-630. [CrossRef]

39. Wang, L.-L.; Gu, Y. Efficient Dual Algorithms for Image Segmentation Using TV-Allen-Cahn Type Models. Commun. Comput. Phys. 2011, 9, 859-877. [CrossRef]

40. Schonlieb, C.B.; Bertozzi, A. Unconditionally stable schemes for higher order inpainting. Commun. Math. Sci. 2011, 9, 413-457.

41. Ruuth, S.J. Implicit-explicit methods for reaction-diffusion problems in pattern formation. J. Math. Biol. 1995, 34, 148-176. [CrossRef]

42. Craus, M.; Paval, S.-D. An Accelerating Numerical Computation of the Diffusion Term in a Nonlocal Reaction-Diffusion Equation. Mathematics 2020, 8, 2111. [CrossRef] 